\title{
Improving transit characterisation with Gaussian process modelling of stellar variability
}

\author{
S. C. C. Barros ${ }^{1}$, O. Demangeon ${ }^{1}$, R. F. Díaz ${ }^{2,3}$, J. Cabrera ${ }^{4}$, N. C. Santos ${ }^{1,5}$, J. P. Faria ${ }^{1,5}$, and F. Pereira ${ }^{1,5}$ \\ ${ }^{1}$ Instituto de Astrofísica e Ciências do Espaço, Universidade do Porto, CAUP, Rua das Estrelas, 4150-762 Porto, Portugal \\ e-mail: susana.barros@astro.up.pt \\ 2 Facultad de Ciencias Exactas y Naturales, Universidad de Buenos Aires, Buenos Aires, Argentina \\ ${ }^{3}$ CONICET - Universidad de Buenos Aires, Instituto de Astronomía y Física del Espacio (IAFE), Buenos Aires, Argentina \\ ${ }^{4}$ Deutsches Zentrum fur Luft- und Raumfahrt, Rutherfordstr. 2, 12489 Berlin, Germany \\ ${ }^{5}$ Departamento de Fisica e Astronomia, Faculdade de Ciencias, Universidade do Porto, Rua Campo Alegre, 4169-007 Porto, Portugal
}

Received 13 June 2019 / Accepted 31 December 2019

\begin{abstract}
Context. New photometric space missions to detect and characterise transiting exoplanets are focusing on bright stars to obtain high cadence, high signal-to-noise light curves. Since these missions will be sensitive to stellar oscillations and granulation even for dwarf stars, they will be limited by stellar variability. Therefore, it is crucial and timely to develop robust methods to account for and correct for stellar variability.

Aims. We tested the performance of Gaussian process (GP) regression on the characterisation of transiting planets, and in particular to determine how many components of variability are necessary to describe high cadence, high signal-to-noise light curves expected from CHEOPS and PLATO. To achieve this, we selected a sample of bright stars observed in the asteroseismology field of CoRoT at high cadence (32 s) and high signal-to-noise ratio (S/N).

Methods. We used GPs to model stellar variability including different combinations of stellar oscillations, granulation, and rotational modulation models. We preformed model comparison to find the best activity model fit to our data. We compared the best multicomponent model with the usual one-component model used for transit retrieval and with a non-GP model.

Results. We found that the best GP stellar variability model contains four to five variability components: one stellar oscillation component, two to four granulation components, and/or one rotational modulation component, which is consistent with results from asteroseismology. However, this high number of components is in contrast with the one-component GP model (1GP) commonly used in the literature for transit characterisation. Therefore, we compared the performance of the best multi-component GP model with the 1GP model in the derivation of transit parameters of simulated transits. We found that for Jupiter- and Neptune-size planets the best multi-component GP model is slightly better than the 1GP model, and much better than the non-GP model that gives biased results. For Earth-size planets, the 1GP model fails to retrieve the transit because it is a poor description of stellar activity. The non-GP model gives some biased results and the best multi-component GP is capable of retrieving the correct transit model parameters.

Conclusions. We conclude that when characterising transiting exoplanets with high S/Ns and high cadence light curves, we need models that couple the description of stellar variability with the transits analysis, like GPs. Moreover, for Earth-like exoplanets a better description of stellar variability (achieved using multi-component models) improves the planetary characterisation. Our results are particularly important for the analysis of TESS, CHEOPS, and PLATO light curves.
\end{abstract}

Key words. planets and satellites: fundamental parameters - planets and satellites: composition - methods: data analysis techniques: photometric - stars: activity

\section{Introduction}

Observations of exoplanets are affected by stellar variability. In general the impact is higher for radial velocity observations; as transit signals are localised in time, they can often be separated relatively easily from the stellar variability (e.g. CoRoT-7b; Léger et al. 2009; Queloz et al. 2009; Haywood et al. 2014; Barros et al. 2014 ). However, as the precision of transit observations increases, stellar intrinsic variability will become the dominant limitation in transit observations of exoplanets, especially for small planets whose transit depths can be of the order of the amplitudes of stellar variability (an Earth-size planet orbiting a Sun-like star has a transit depth of $\sim 80 \mathrm{ppm}$ ). The shorter ingress and egress time for Earth-size planets implies that the shape will be more affected by short timescale stellar variability.
Stellar variability has different origins and covers a wide range of timescales. Stars with convective envelopes show $p$-mode oscillations with periods of a few minutes and amplitudes $\sim 10 \mathrm{ppm}$ in solar-type stars (Kjeldsen \& Bedding 1995; Kallinger et al. 2014). These stars also show photometric variability due to granulation and super-granulation. Granulation has timescales from $\sim 20$ min up to days and amplitudes reaching a few hundred ppms (Kallinger et al. 2014; Meunier et al. 2015). The effect of this low amplitude short timescale variability in transit observations has been poorly studied until now. In contrast, one variability effect that has been well studied is the rotational modulation due to magnetic activity features on the stellar surface, like spots, flares, and plages. These give rise to much higher photometric variations reaching $\sim 1000 \mathrm{ppm}$ on a timescale corresponding to the rotational period of the star 
(Lagrange et al. 2010). Although the amplitude of these variations can be high in active stars, their timescale is much longer than the transit timescale, and hence their effect on the transit shape can be corrected by detrending locally with a first- or second-order polynomial. However, a residual ambiguity in the determination of the absolute out-of-flux level can lead to differences in the determination of the planetary radius (Czesla et al. 2009) as seen, for example, in the case of WASP-10b (Christian et al. 2009; Maciejewski et al. 2011; Barros et al. 2013). Without resolving the stellar surface the only way to mitigate this effect is to have very long baselines of observations. On longer timescales (years), stars vary due to the stellar magnetic cycle that leads to long-term evolution of spot coverage and produces flux variations up to a few percent (Baliunas et al. 1995; Hall et al. 2007; Lovis et al. 2011).

In this work we address how to recover accurate and precise planetary parameters for transiting planets in presence of stellar variability levels representative of current and future spaceborne missions. We investigate the effect of stellar oscillations and granulation in transit observations of exoplanets using the framework of Gaussian processes (GPs). We test several models that include different components of stellar variability including stellar oscillations, granulation, and rotation. The advantages of using GPs to model stellar rotational modulation in radial velocity observations of exoplanets has been shown in many works. For example, Haywood et al. (2014) and Faria et al. (2016) showed that it is possible to correct the stellar rotational modulation, and to detect planetary signals that are much smaller than the stellar activity signal. It was also shown that modelling stellar activity with GPs in photometric observations of exoplanets allows us to correct for these factors (e.g. Aigrain et al. 2015; Serrano et al. 2018). However, the stellar rotational modulation signal is quasi-periodic and since we can sample the typical timescale with enough data it is possible to make good predictions. In contrast, granulation is a stochastic process and making predictions is much harder. The stellar oscillations are also quasi-periodic, but contain many modes which might lead to worse predictions. Hence, it is not clear whether a combined model of the different variability components will improve transit parameter determination. We expect that the combined model will account for the uncertainty introduced by stellar variability increasing the accuracy of the parameters even at the expense of precision. Perhaps more interesting is whether it can correct stellar variability and increase the precision of the parameters. Another question we address is how many components of stellar variability are necessary to model the light curves.

Photometric variability due to stellar oscillations and granulation was detected in Kepler observations (Borucki et al. 2010; Mathur et al. 2011; Bastien et al. 2014; Cranmer et al. 2014; Kallinger et al. 2014). However, these were relatively rare due to the long cadence of Kepler light curves and average magnitude of the Kepler field stars. Hence, to study how stellar oscillations and granulation affect transit observations, we chose bright stars observed in the asteroseismology field of CoRoT (Baglin et al. 2006). We want to test whether GPs can correct or account for stellar variability in high cadence high signal-to-noise light curves, and to determine the best model to use. We start by presenting our sample in Sect. 2, followed by the presentation of our variability model and model comparison methods in Sect. 3. In Sect. 4 we show how we derived the best stellar variability GP model. In Sect. 5 we determine in which cases the multicomponent model is required. Finally, we discuss the implication of our results in Sect. 6.

\section{Stellar sample}

\subsection{CoRot light curves}

The CoRoT satellite had two science channels for its two science goals: asteroseismology and exoplanet search. Each initially had two CCDs, which were reduced to one CCD per field after the failure of the Data Processing Unit 1 on 8 March 2009. Due to pointing restrictions CoRoT observations were divided into long runs with a duration up to 150 days and short runs with a duration of $\sim 30$ days.

In the exoplanet field, to increase the probability of detecting transiting exoplanets 6000-12000 target stars (mag $V>9$ ) were monitored in each run, the majority with a cadence of 512 s. In the asteroseismology channel to reach the signal-tonoise ratio $(\mathrm{S} / \mathrm{N})$ necessary to detect stellar oscillations, only a few very bright stars were observed (average $V$ magnitude of 7) at a much higher sampling rate $(1 \mathrm{~s})$. The asteroseismology channel had five stellar windows $(50 \times 50$ pixels $)$, five sky-reference windows, and two offset reference windows per CCD. Aperture photometry was performed on board using a mask optimised for the position in the CCD. Several corrections to the data were applied to correct for instrumental effects, and the data was resampled to $32 \mathrm{~s}$ in the heliocentric frame. In particular, several steps of outlier rejection were preformed to clean cosmic ray hits, first in the images and then in the light curves. We used the latest reduction of the light curves that is available through the CoRoT legacy archive ${ }^{1}$. No extra outlier rejection was preformed. A full description of the CoRoT satellite can be found in Auvergne et al. (2009), while a more recent review can be found in CoRot Team (2016).

Since we are interested in observations at high cadence and with a very high $\mathrm{S} / \mathrm{N}$, we selected observations made by the asteroseismology field of CoRoT during long runs. Our sample contains three main sequence stars with magnitudes between 5.7 and 6.3 and photometric precision between 56 and 84 ppm over $32 \mathrm{~s}$ bin, and a slightly fainter sub-giant star $(\operatorname{mag}=8.0)$ for comparison. This sample allowed us to probe the timescales and amplitudes that we are interested in. We included targets with known non-transiting planets so that our sample is representative of planet hosts. The properties of our four stars are given in Table 1, while the details of the observations are given in Table 2. In this table we also give the combined differential photometric precision (CDPP)-6.5 h calculated following the method of Gilliland et al. (2011) for comparison with the Kepler sample. The CDPP-6.5 only measures the intrinsic variability of stars on timescales between $6.5 \mathrm{~h}$ and 2 days. For all the dwarfs in our sample we obtained a CDPP-6.5 h lower than $5 \mathrm{ppm}$. Hence, our stars have low intrinsic variability when compared with Kepler dwarfs stars (Gilliland et al. 2011) and also when compared to the Sun. Therefore, these low variability stars are representative of the best targets for transit search with future missions. For the sub-giant star HD 179079 the CDPP-6.5 $\mathrm{h}$ is higher, as expected, (11.2 ppm) and will be used as comparison.

\subsection{Previous asteroseismology analysis}

As main targets of the CoRoT asteroseismology program, results of the asteroseismic analysis of most of these stars were already reported in the literature. The light curve of HD 43587 was analysed by Boumier et al. (2014) who measured $p$-mode oscillations with frequency peaking at $2247 \pm 15 \mu \mathrm{Hz}(7.42 \mathrm{~min})$. The light

1 https://corot.cnes.fr/en/release-corot-legacy-data 
S. C. C. Barros et al.: Improving transit characterisation with Gaussian process modelling of stellar variability

Table 1. Characteristics of the sample of stars: spectral type, $V$ magnitude, rotation period $\left(P_{\text {rot }}\right)$, and presence of a known planet.

\begin{tabular}{llcll}
\hline \hline Star & Spectral type & $V$ mag & $P_{\text {rot }}$ & Known planet \\
\hline HD 43587 & G0 V & 5.7 & Long & \\
HD 49933 & F3 V & 5.8 & 3.4 & \\
HD 52265 & G0 V & 6.3 & 12.3 & Non-transiting Jupiter \\
HD 179079 & G5 IV & 8.0 & & Non-transiting warm Neptune \\
\hline
\end{tabular}

Table 2. Details of the observations of each star including the name of the CoRoT run, the total duration of the observations, and the uncertainties per 32-s bin.

\begin{tabular}{llccc}
\hline \hline Star & Run & $\begin{array}{c}\text { Duration } \\
(\text { days })\end{array}$ & $\begin{array}{c}\sigma(/ 32 \mathrm{~s}) \\
(\mathrm{ppm})\end{array}$ & $\begin{array}{c}\text { CDPP 6.5 h } \\
(\mathrm{ppm})\end{array}$ \\
\hline HD 43587 & LRa03 & 142 & 56 & 4.3 \\
HD 49933 & IRa01 & 61 & 57 & 4.4 \\
HD 49933 & LRa01 & 137 & 56 & 4.1 \\
HD 52265 & LRa02 & 117 & 84 & 4.6 \\
HD 179079 & LRc09 & 55 & 170 & 11.2 \\
\hline
\end{tabular}

curve of HD 49933 was analysed by Appourchaux et al. (2008) and Benomar et al. (2009) who measured solar-type oscillations with a central frequency of $1760 \mu \mathrm{Hz}(9.50 \mathrm{~min})$ and a rotation period of 3.4 days. The light curve of HD 52265 was analysed by Ballot et al. (2011) who reported solar-type oscillations in the range $1500-2550 \mu \mathrm{Hz}$ with a central frequency of $2090 \pm 20 \mu \mathrm{Hz}$ (7.97 $\mathrm{min}$ ). They also reported one granulation component with a period of $4.03 \pm 0.03 \mathrm{~min}$ and rotational modulation with a period of $12.3 \pm 0.15$ days with signs of differential rotation. No analysis was published from HD 179079 CoRoT observations.

\section{Variability modelling and model comparison}

\subsection{Gaussian process regression}

Gaussian processes (GPs) are non-parametric models that are useful for cases where the functional form of the model is not known a priori (Rasmussen \& Williams 2006). GP models have been used for Bayesian regression to model instrumental systematic noise (Gibson et al. 2012) and stochastic processes. Recently they have been also used to model stellar activity (Haywood et al. 2014; Aigrain et al. 2015). The form of a GP is defined by a mean function and a covariance matrix, which is modelled by a kernel function. There are several classes of GP kernels which describe different behaviour for the correlation between data points. An advantage of the Bayesian framework is that it penalises complex models and hence avoids overfitting.

One disadvantage of GPs is that the computation time generally scales with the number of observations cubed $\left(O\left(n^{3}\right)\right)$. Fortunately, a new implementation of GPs has recently been developed called celerite (Foreman-Mackey et al. 2017), which considerably speeds up computation time as it scales with $O n$. This implementation comes with some restrictions; for example, it can only be applied to one-dimensional datasets and requires stationary processes. This means that the kernels are required to be functions of $\tau$ alone, with $\tau_{i j}=\left|t_{i}-t_{j}\right|$. It also requires the kernels to be a mixture of exponential functions. Foreman-Mackey et al. (2017) show that they can be re-written as a mixture of quasi-periodic oscillators. Furthermore, what is most interesting for our case is that some of the possible celerite kernels are well suited to describe different forms of stellar variability. According to Eq. (49) in Foreman-Mackey et al. (2017), the kernel for a stochastically driven, damped harmonic oscillator with a quality factor $(Q)$ larger than 0.5 is given by

$$
\begin{aligned}
k\left(\tau ; S_{0}, Q, \omega_{0}\right)= & S_{0} \omega_{0} Q e^{-\frac{\omega_{0} \tau}{2 Q}} \cos \left(\eta \omega_{0} \tau\right) \\
& +\frac{1}{2 \eta Q} \sin \left(\eta \omega_{0} \tau\right),
\end{aligned}
$$

where $\omega_{0}$ is the frequency of the undamped oscillator, $S_{0}$ is related to the power spectral density (PSD) at $\omega=\omega_{0}$ by $S_{0}=$ $\operatorname{PSD}\left(\omega_{0}\right) /\left(\sqrt{2 / \pi} Q^{2}\right)$, and $\eta=\left|1-\left(4 Q^{2}\right)^{-1}\right|^{1 / 2}$.

For the particular case of $Q=1 / \sqrt{2}$ this kernel has the same power spectrum density as stellar granulation (Harvey 1985; Kallinger et al. 2014) and can be rewritten as

$k(\tau)=S_{0} \omega_{0} e^{-\frac{1}{\sqrt{2}} \omega_{0} \tau} \cos \left(\frac{\omega_{0} \tau}{\sqrt{2}}-\frac{\pi}{4}\right)$

(Eq. (51) in Foreman-Mackey et al. 2017). Therefore, we use this kernel to describe stellar granulation and we refer to it as the granulation kernel. In the classical GP framework this is close to the square exponential kernel which was previously used to model stellar activity together with transit modelling (e.g. Dawson et al. 2014; Barclay et al. 2015).

For the limit of $Q>1$, the kernel given by Eq. (1) has a Lorentzian power spectrum density near the peak frequency. Therefore, it can be used to describe stellar oscillations. We also use this model and we refer to it as the oscillation kernel.

To model stellar variability due to rotation modulation of spots and plages it is common to use the quasi-periodic kernel in radial velocity modelling (Haywood et al. 2014) and in photometry (Aigrain et al. 2015; Serrano et al. 2018). A kernel with a similar covariance function in celerite was proposed by Foreman-Mackey et al. (2017; Eq. (61)),

$k(\tau)=\frac{B}{2+C} e^{-\tau / L}\left[\cos \left(\frac{2 \pi \tau}{P_{\text {rot }}}\right)+(1+C)\right]$,

where $P_{\text {rot }}$ is the rotation period of the star and $B>0, C>0$, and $L>0$. We use this kernel to model stellar rotation modulation in the light curves, which we refer to as the rotation kernel.

The objective of this study is to test which of these variability components can be detected in our light curves, and to determine their significance. To test this, we construct several noise models that include different components of granulation, oscillation, and rotation by adding the respective covariance matrixes (kernels) described above. We call them noise models because they model the covariance and not directly the data. In our case the deterministic model is zero in the first part of this work (Sect. 4), while for the second part it is the transit model (Sect. 5). The parameters of the GP are called hyper-parameters to distinguish 
them from the transit model parameters. The different combinations of the noise models considered will be presented in Sect. 4. For each noise model, we find the best hyper-parameters for each light curve maximising the log-likelihood function

$\ln \mathcal{L}(\mathbf{r})=-\frac{1}{2} \mathbf{r}^{\mathrm{T}}\left(\mathbf{K}+\sigma_{i}^{2} \mathbf{I}\right)^{-1} \mathbf{r}-\frac{1}{2} \ln \left(\left|\mathbf{K}+\sigma_{i}^{2} \mathbf{I}\right|\right)-\frac{n}{2} \ln (2 \pi)$,

where $\mathbf{r}$ are the residuals obtained by subtracting the deterministic model to the data, $|\mathbf{A}|$ is the determinant of the matrix A, and $\mathrm{n}$ is the number of data points. The term $\sigma^{2} \mathbf{I}$ represents an additional white noise component, where $\mathbf{I}$ is the identity matrix and $\sigma^{2}$ is the variance of the extra noise.

We find the maximum of the log-likelihood function using the Markov chain Monte Carlo (MCMC) algorithm emcee (Foreman-Mackey et al. 2013). We used 32 chains, which is double the maximum number of parameters fitted (16). Each chain has 10000 iterations. We separated the exploration in three stages. The first stage was used to explore the parameter space and find the global maximum and consisted of 2000 steps. The chains were started at random points from the prior. The second stage was used to consolidate the global maximum and consisted of 4000 steps. We started the chains close to the parameter set with highest posterior probability found in the first stage. The third stage was used to explore the parameter space next to the global maximum and derive the best hyper-parameters and their uncertainties. It was just a continuation of the second stage, but only this last stage was kept and analysed. Convergence was checked with the Geweke algorithm (Geweke 1992) and when necessary some residual burn-in was discarded from the third stage. The chains were combined in a master chain that was used for further analysis. To infer the parameter values we used the median of the master chain distributions for all the hyperparameters except for the stellar rotation period. The median is usually a better estimator than the mode, which is very sensitive to bin size. However, for distributions with large tails and high asymmetry (in our case the stellar rotation period) the median is a poor estimator and the mode is better. Hence, in these particular cases we used the mode of the distribution. In some cases the stellar rotation period could only be constrained to be above a certain value, and in these cases we quote the $3 \sigma$ limit. The uncertainties of the hyper-parameters were estimated from the 16 th and 84th percentiles of the chains.

For the GP noise models, we used wide priors for the hyper-parameters. For comparison with asteroseismology, we converted the hyper-parameters of Eqs. (1) and (2) into the parameters usually used in asteroseismology analysis to fit the power spectrum following Pereira et al. (2019):

$a_{\text {gran }}=\sqrt{\sqrt{2} S_{0} \omega_{0}}$,

$a_{\mathrm{osc}}=4 S_{0} Q^{2}$,

$\tau=\frac{2 \pi}{\omega_{0}}$.

The priors are the same for each component of variability (i.e. no order was imposed on the granulation timescales). This was meant to simplify the exploration of the parameter space. In cases where the second component of granulation had the same period as the first within the errors, we considered that the second component was not needed. The priors used for the oscillation kernel and the rotation kernel are given in Tables 3 and 4. The granulation kernel is a particular case of the oscillation kernel where $Q=1 / \sqrt{2}$ and the rest of the hyper-parameters have the same priors as the oscillation kernel.
Table 3. Priors for the granulation and oscillation kernels.

\begin{tabular}{lc}
\hline \hline Parameter & Prior \\
\hline $\ln$ white noise & $\mathcal{U}(-15 ; 5)$ \\
$\ln S_{0}$ & $\mathcal{U}(-5 ; 25)$ \\
$\ln \omega_{0}$ & $\mathcal{U}(-1 ; 8)$ \\
$\ln Q$ & $\mathcal{U}(-5 ; 2.35)$ \\
\hline
\end{tabular}

Notes. $\mathcal{U}(a ; b)$ is a uniform probability distribution between $a$ and $b$.

Table 4. Priors for the rotation kernel.

\begin{tabular}{lc}
\hline \hline Parameter & Prior \\
\hline $\ln$ white noise & $\mathcal{U}(-15 ; 5)$ \\
$\ln a$ & $\mathcal{U}(7 ; 12)$ \\
$\ln b$ & $\mathcal{U}(-1 ; 0)$ \\
$\ln c$ & $\mathcal{U}(-4.5 ; 0.5)$ \\
$\ln$ period & $\mathcal{U}(0.5 ; 5)$ \\
\hline
\end{tabular}

Notes. $\mathcal{U}(a ; b)$ is a uniform probability distribution between $a$ and $b$.

\subsection{Model comparison}

Bayesian probability theory allows us to perform model comparison by the computation of the odds ratio between two hypotheses (e.g. Díaz et al. 2014). The odds ratio for a pair of hypotheses is the multiplication between the prior odds and the Bayes factor. The prior odds are the a priori probability of each model. In our study we assume that the prior odds are equal for all the models as different stars are dominated by different types of variability and the noise of the data will affect the detection of the variability components in a way not known a priori. Therefore, in our case, the odds ratio is equal to the Bayes factor, which is the ratio of the two evidence terms. The evidence of a model is given by the integral of the joint posterior of the model's parameters

$p\left(D \mid H_{\mathrm{i}}, I\right)=\int p\left(D \mid \theta_{\mathbf{i}}, H_{\mathrm{i}}, I\right) \cdot \mathrm{d} \theta_{\mathbf{i}} \cdot \operatorname{prior} \theta_{\mathbf{i}} H_{i}, I$,

where $D$ represents the data, $H_{\mathrm{i}}$ the hypothesis $\mathrm{i}, I$ the prior information, $\theta_{\mathbf{i}}$ the parameter vector of the model associated with hypothesis $H_{i}$, prior $\theta_{\mathbf{i}} H_{\mathrm{i}}, I$ the joint prior distribution, and $p\left(D \mid \theta_{\mathbf{i}}, H_{\mathrm{i}}, I\right)$ the likelihood for a given dataset $D$ under the assumption of hypothesis $H_{\mathrm{i}}$. Bayesian model comparison penalises models with a larger number of parameters because they dilute the normalised prior density, leading to a natural occam's razor.

The priors are given in Tables 3 and 4, and the likelihood is computed with Eq. (4). This multi-dimensional integral is in general impossible to compute analytically, and several approximations are used. In our case we used importance sampling (Kass \& Raftery 1995) to approximate the integral, and in particular the Perrakis method (Perrakis et al. 2014). This method uses samples of marginal posterior probability distributions from an MCMC algorithm to estimate the evidence.

To estimate the evidence we used 3000 independent samples of the posterior probability distribution. For each model this procedure was repeated 400 times in order to obtain the distribution of the estimator of the evidence. The value of the evidence we quoted is the median of this distribution. More details about our computation of the evidence can be found in Díaz et al. (2014) and Nelson et al. (2018). 
S. C. C. Barros et al.: Improving transit characterisation with Gaussian process modelling of stellar variability
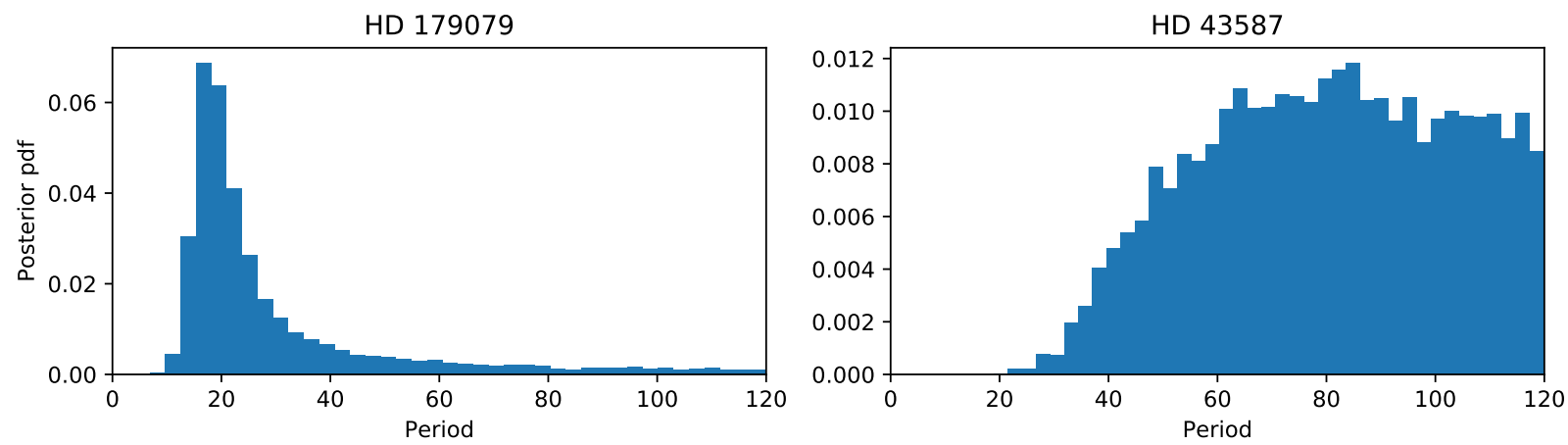

Fig. 1. Derived posterior probability distributions for the stellar rotation period for two examples: HD 179079 where the rotation period is well determined, and HD 43587 where the rotation period is longer than 29.13 days at $3 \sigma$.

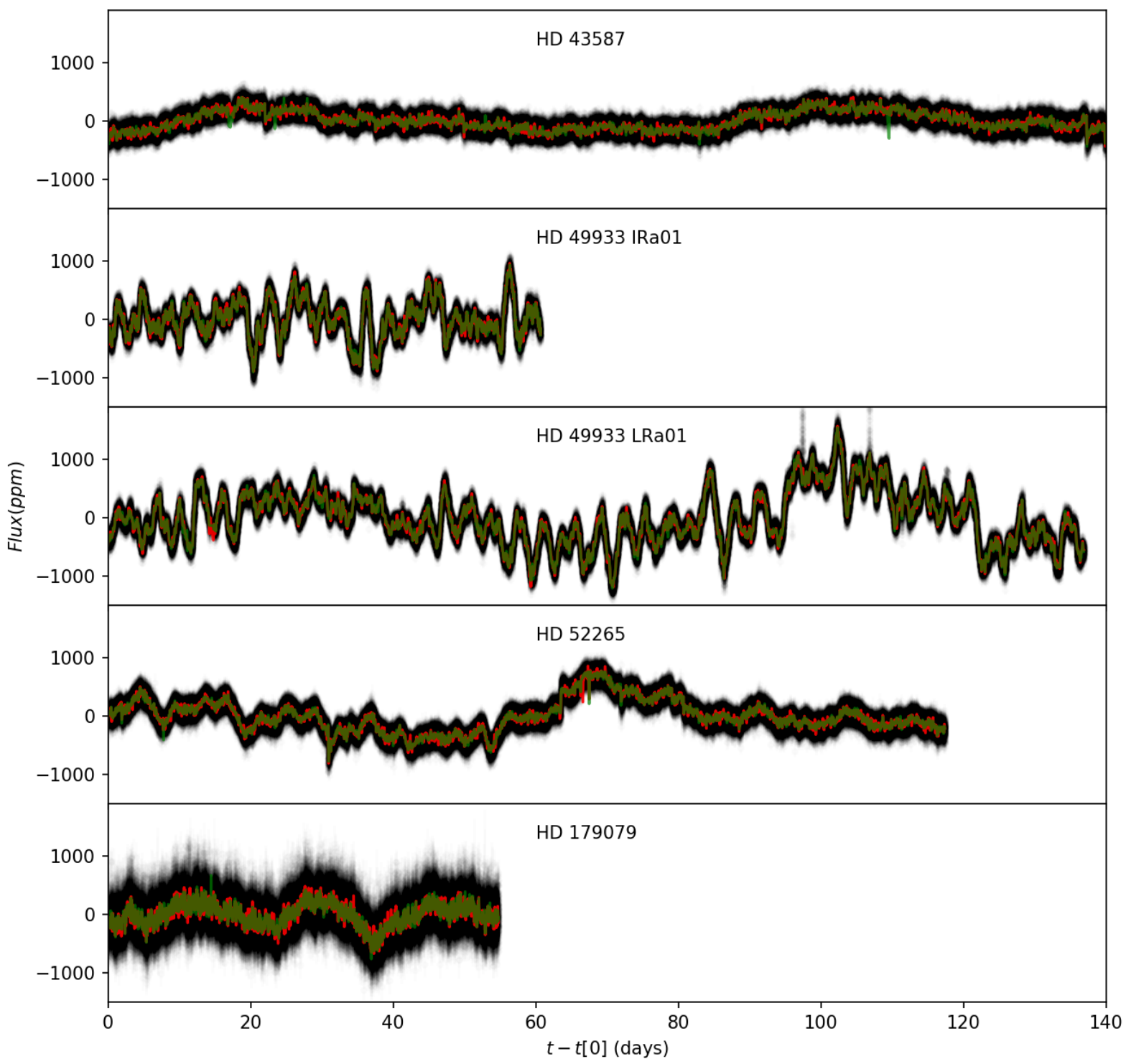

Fig. 2. Light curves of all our sample stars overplotted with the best GP model in red and the G1 model in green. For clarity we decreased the transparency of the light curve points. The $x$ and $y$ axes are the same for all the observations for easier comparison between the different observations.

\subsection{Stellar rotation}

From the light curves (Figs. 2 and 3) it is evident that HD 49933 and HD 52265 show a clear sign of rotation spot modulation. This implies that some light curves require a model that includes a rotation kernel. Preliminary tests showed that it was difficult to fit the stellar rotation using our rotation celerite kernel (Eq. (3)) due to the strong short-term variability present in the light curves that is also reproduced by this Kernel. This was solved once more kernels were added to the GP model to account for 


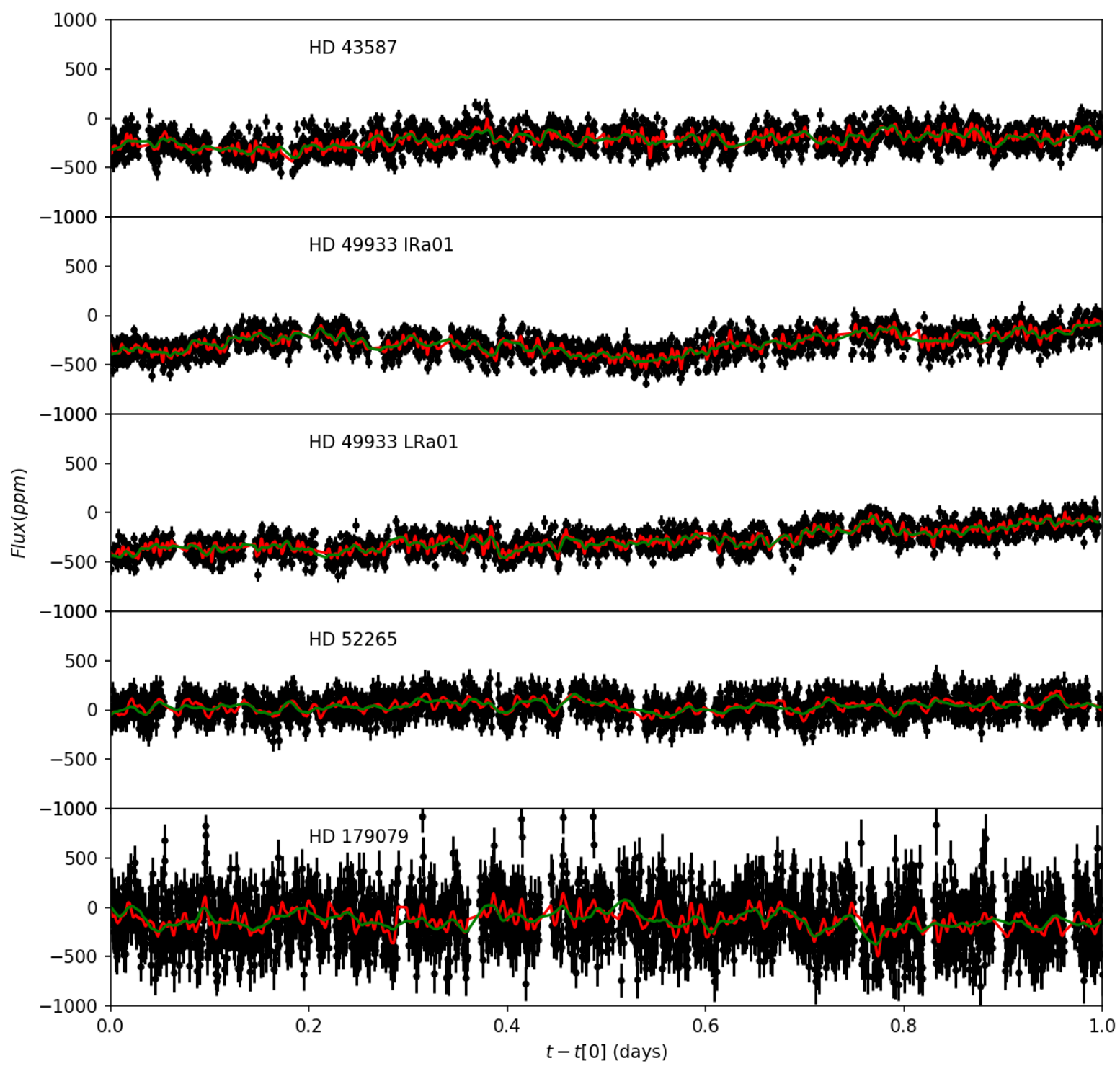

Fig. 3. Zoom of the first day of observations of all our sample stars shown in Fig. 2. In the zoom it is clearer that the best model (red) has a much higher frequency component than the G1 model (green).

short-term variability. However, we found that for clarity it was easier to start by dividing our sample into stars with and without a measurable rotation period.

To determine for which light curves it was possible to measure the rotation period we averaged out high frequency variations by binning the light curves into two-hour bins. The binned light curves were fitted with the method described above with one rotation kernel. The derived rotation period for each of the observation is given in Table 5 . We derived the rotation period for HD 49933, HD 52265, and HD 179079. However, we could only put a lower limit on the rotation period of HD 43587.

The rotation periods were already derived from these light curves by Appourchaux et al. (2008) and Benomar et al. (2009) for HD 49933, and by Ballot et al. (2011) for HD 52265, as mentioned in Sect. 2.2. Our values are in agreement with the published values. In Fig. 1 we show the posterior probability distribution of the rotation period for two examples, one where we can determine the rotation period (HD 179079) and one where we can only derive a lower limit (HD 43587). It should be noted that for HD 49933 the derived rotation period for the two observations agrees well. Since we could not determine the rotation period for HD 43587, for this star we did not consider
Table 5. Derived stellar rotation period for the binned light curves.

\begin{tabular}{lc}
\hline \hline Star & $\begin{array}{c}\text { Rotation period } \\
\text { (days) }\end{array}$ \\
\hline HD 43587 & $>29.13$ \\
HD 49933 (IRa01) & $3.19 \pm 0.16$ \\
HD 49933 (LRa01) & $3.294 \pm 0.082$ \\
HD 52265 & $10.3 \pm 1.2$ \\
HD 179079 & $17.8_{-1.6}^{+29}$ \\
\hline
\end{tabular}

models that include a rotation kernel for the subsequent analysis. Hence, we separated HD 43587 from the main group of stars for which we detect the rotation period.

\section{What is the best stellar variability GP model?}

\subsection{GP modelling}

All the light curves were fitted with a set of models combining granulation, oscillation, and rotation when needed (see 
Table 6. Differences in the $\log$ evidence of each model relative to the best GP noise model for targets with measured stellar rotation period.

\begin{tabular}{lcccc}
\hline \hline Model & HD 49933 IRa01 & HD 49933 LRa01 & HD 52265 & HD 179079 \\
\hline G1 & -6776 & -18489 & -11223 & -1768 \\
G2 & -786 & -2270 & -1514 & -222 \\
G3 & -161 & -478 & -344 & -130 \\
G4 & -125 & -240 & -152 & -17 \\
G5 & -112 & -211 & -144 & -18 \\
G1R1 & -251 & -574 & -845 & -125 \\
G2R1 & -121 & -188 & -137 & -18 \\
G3R1 & -112 & -181 & -136 & -13 \\
G2R1O1 & 0 & 0 & 0 & 0 \\
G3O1 & -9 & -84 & -56 & -6 \\
G4O1 & - & - & -16 & - \\
\hline
\end{tabular}

Sect. 3.3). The notation used to designate a model summarises the number of granulation, oscillation, and rotation kernels used for a given model. We used the letter $\mathrm{G}$ for granulation, $\mathrm{O}$ for oscillation, and $\mathrm{R}$ for rotation. The number following the letter indicates how many of these kernels are considered. For example, the G5 model is composed of 5 distinct granulation kernels, the G4O1 model is composed of 4 granulation kernels and 1 oscillation kernels.

As we can build an infinite number of models from our three building blocks, we limited our set of models according to the following criteria:

- We limited the total amount of components to five according to the maximum number of components considered in the literature (Harvey 1985; Corsaro et al. 2015). This limits the computational time to a considerable but still manageable amount (33 days per target).

- We limited the number of oscillation kernels to one since we expected only one oscillation component in the light curves.

- We limited the number of rotation kernels to one since we expected only one rotation component in the light curves.

- We did not limit the maximum number of granulation components to three, as expected, because we assumed this kernel is also capable of detecting other types of variability (instrumental or stellar), and we considered it to be the simpler model as it has fewer parameters. The classical squared exponential kernel (which is the one closer to the granulation celerite kernel) is the most commonly used in the literature to model both instrumental and stellar red noise in light curves. We started by considering models made only with granulation components (up to five). We then replaced some of these components by oscillation or rotational components.

The final sets of models that were considered are given in Tables 6 and 7 for the stars with and without detected rotational modulation respectively. Tables 6 and 7 also present the differences between the logarithm of the evidence (Sect. 3.2) of the best model and all the other models considered. We analysed the fits for convergency and made sure that the components were different. When two components effectively had the same timescale the fit was not considered for model comparison. This was the case of the model G4O1 for both light curves of HD 49933 and the light curve of HD 179079, where one of the components had the same timescale as another component.

To identify the best model, we use the classical criteria of Jeffreys (1998) stating that a more complex model is only considered as better if it is associated with evidence 150 times
Table 7. Difference in the log evidence of each model relative to the best GP noise model the target with unmeasured stellar rotation period.

\begin{tabular}{lc}
\hline \hline Model & HD 43587 \\
\hline G1 & -11256 \\
G2 & -1847 \\
G3 & -652 \\
G4 & -369 \\
G5 & -345 \\
G3O1 & -66 \\
G4O1 & 0 \\
\hline
\end{tabular}

higher than the simpler model, which corresponds to a delta $\log$ evidence superior to five. According to this threshold the best GP noise models are G2R1O1 for all of the light curves with detected rotational modulation (HD 49933, HD 52265, and HD 179079) and G4O1 for HD 43587. It is worth noting that the results are the same for the two light curves of HD 49933. Importantly, the difference between the best GP noise model and the GP noise model with just one kernel (G1), which is usually used in the literature, is highly significant. This implies that a multi-component model is really needed when modelling the variability of these stars. Given that it is common practice to use just one kernel to model the photometric stellar variability in transit parameter retrieval, it is interesting to further compare the best GP noise model we found with the G1 model. In Fig. 2 we show the full light curves for each star and we overplot the best model (red) and the G1 model (green). In Fig. 3, we show a oneday zoom of the previous figure so that the difference between models on the shorter timescales is clearer). From the figures it is evident that the G1 model reproduces well the long-term variability of the light curves, but does not reproduce the short-term variability.

In Table 8 we give the fitted hyper-parameters for all the light curves when we considered the G1 model, and in Tables 9 and 10 we give the fitted hyper-parameters for the best GP noise model. The timescales found for the G1 model are between 20 and $40 \mathrm{~min}$. The timescales found for the best model are close to $8 \mathrm{~min}$ for the oscillations (14 min for the sub-giant HD 179079), 7-27 min for the first granulation kernel (43 min for the sub-giant HD 179079), and up to $14 \mathrm{~h}$ for the longer period granulation kernel. In general, the amplitudes of variability are higher for the rotational component as expected.

\subsection{Comparison with previous asteroseismology analysis}

In order to validate our method we compared our results with previous results from asteroseismology. The majority of the previous analysis of these light curves using frequency domain methods only report the timescales of the stellar oscillations. Stellar oscillations were found for all of the light curves previously analysed. In our analysis, models that include one oscillation kernel were strongly preferred for all the stars, and hence we also detect stellar oscillations in all the light curves.

CoRoT light curves have gaps due to the crossing of the South Atlantic anomaly. Since our method uses GPs that are applied in the time domain, it is not affected by gaps contrary to frequency domain analysis. For asteroseismic analysis, it is usual to fill these gaps in order to apply the Fourier domain methods. Since even the best interpolation methods will alter the data, we do not perform gap filling in our analysis. 
Table 8. Derived hyper-parameters for the G1 model.

\begin{tabular}{lccc}
\hline \hline Star & wn & $\begin{array}{c}\text { Amplitude } \\
\text { ppm }\end{array}$ & $\begin{array}{c}\text { Period } \\
\text { min }\end{array}$ \\
\hline HD 43587 & $4.2356 \pm 0.0022$ & $209.1 \pm 2.2$ & $20.97 \pm 0.30$ \\
HD 49933 (IRa01) & $4.0466 \pm 0.0040$ & $438.0 \pm 8.5$ & $32.22 \pm 0.65$ \\
HD 49933 (LRa01) & $3.9596 \pm 0.0029$ & $627.8 \pm 8.5$ & $35.45 \pm 0.46$ \\
HD 52265 & $3.9284 \pm 0.0053$ & $384.6 \pm 6.1$ & $39.91 \pm 0.74$ \\
HD 179079 & $5.1370 \pm 0.0039$ & $258.4 \pm 4.8$ & $24.87 \pm 0.76$ \\
\hline
\end{tabular}

Table 9. Derived G2R1O1 model hyper-parameters.

\begin{tabular}{|c|c|c|c|c|c|c|c|c|c|}
\hline \multirow[t]{2}{*}{ Star } & \multicolumn{2}{|c|}{ Rotation kernel } & \multicolumn{3}{|c|}{ Oscillation kernel } & \multicolumn{2}{|c|}{ First granulation kernel } & \multicolumn{2}{|c|}{ Second granulation kernel } \\
\hline & $\begin{array}{l}\text { Amplitude } \\
\text { ppm }\end{array}$ & $\begin{array}{c}\text { Rotation period } \\
\text { days }\end{array}$ & $\begin{array}{l}a_{\mathrm{osc}} \\
\mathrm{ppm}\end{array}$ & $\begin{array}{l}\tau_{\mathrm{osc}} \\
\min \end{array}$ & $\mathrm{q} 1$ & $\begin{array}{c}a_{\text {gran1 }} \\
\text { ppm }\end{array}$ & $\begin{array}{c}\tau_{\text {gran } 1} \\
\min \end{array}$ & $\begin{array}{c}a_{\text {gran2 }} \\
\text { ppm }\end{array}$ & $\begin{array}{c}\tau_{\text {gran2} 2} \\
\min \end{array}$ \\
\hline HD 179079 & $134 \pm 34$ & $17.6 \pm 6.6$ & $25.5 \pm 3.2$ & $13.99 \pm 0.27$ & $2.30 \pm 0.62$ & $104.5 \pm 2.6$ & $42.8 \pm 2.9$ & $53.9 \pm 6.3$ & $588 \pm 89$ \\
\hline HD 49933 (IRa01) & $237 \pm 27$ & $3.23 \pm 0.17$ & $6.46 \pm 0.27$ & $8.687 \pm 0.080$ & $1.80 \pm 0.10$ & $48.5 \pm 1.4$ & $24.8 \pm 1.2$ & $22.1 \pm 4.4$ & $71 \pm 12$ \\
\hline HD 49933 (LRa01) & $293.14 \pm 24.39$ & $3.35 \pm 0.10$ & $6.58 \pm 0.17$ & $8.411 \pm 0.050$ & $1.495 \pm 0.053$ & $50.9 \pm 1.1$ & $26.63 \pm 0.90$ & $32.50 \pm 2.00$ & $97 \pm 13$ \\
\hline HD 52265 & $170 \pm 23$ & $11.6 \pm 2.4$ & $4.83 \pm 0.33$ & $7.491 \pm 0.051$ & $4.48 \pm 0.47$ & $46.7 \pm 1.4$ & $7.40 \pm 0.26$ & $52.12 \pm 0.85$ & $29.73 \pm 0.90$ \\
\hline
\end{tabular}

Notes. The white noise is always between $3.5-5 \mathrm{ppm}$.

Table 10. Derived G5 model hyper-parameters.

\begin{tabular}{|c|c|c|c|c|c|c|c|c|c|c|c|}
\hline \multirow[t]{2}{*}{ Star } & \multicolumn{3}{|c|}{ Oscillation kernel } & \multicolumn{2}{|c|}{ First granulation kernel } & \multicolumn{2}{|c|}{ Second granulation kernel } & \multicolumn{2}{|c|}{ Third granulation kernel } & \multicolumn{2}{|c|}{ Fourth granulation kernel } \\
\hline & $\begin{array}{l}a_{\mathrm{osc}} \\
\mathrm{ppm}\end{array}$ & $\begin{array}{l}\tau_{\mathrm{osc}} \\
\min \end{array}$ & $\mathrm{q} 1$ & $\begin{array}{c}a_{\text {gran1 }} \\
\text { ppm }\end{array}$ & $\begin{array}{c}\tau_{\text {gran } 1} \\
\min \end{array}$ & $\begin{array}{c}a_{\text {gran2 }} \\
\text { ppm }\end{array}$ & $\begin{array}{c}\tau_{\text {gran2}} \\
\min \end{array}$ & $\begin{array}{c}a_{\text {gran3 }} \\
\text { ppm }\end{array}$ & $\begin{array}{c}\tau_{\text {gran3 } 3} \\
\mathrm{~h}\end{array}$ & $\begin{array}{c}a_{\mathrm{gran} 4} \\
\mathrm{ppm}\end{array}$ & $\begin{array}{c}\tau_{\text {gran } 4} \\
\text { days }\end{array}$ \\
\hline HD 43587 & $5.66 \pm 0.20$ & $7.634 \pm 0.037$ & $3.19 \pm 0.15$ & $53.83 \pm 0.76$ & $21.59 \pm 0.59$ & $27.2 \pm 1.4$ & $87 \pm 11$ & $45.2 \pm 1.8$ & $13.98 \pm 0.88$ & $191 \pm 23$ & $15.4 \pm 1.4$ \\
\hline
\end{tabular}

Notes. The white noise is $4.116 \pm 0.002 \mathrm{ppm}$.

However, this might lead to small differences between our analysis and previous asteroseismology results.

Our derived timescales of the stellar oscillations are similar to the published values even if they are not strictly consistent (taking into account error bars). The small differences between the two analyses might be due to specific data reduction procedures, gap filling methods, or the number of components considered to fit the data. A comparison between GPs and asteroseismology methods to analyse stellar granulation and oscillations for red giant stars was preformed by Pereira et al. (2019). They show that both models find the same stellar signals, but there are some slight offsets in the derived parameters due to a difference in the shape of the models used in asteroseismology and the GP kernel used.

For the light curves with detected rotational modulation, GP models that include the rotational kernel are preferred. Our derived rotation periods are in good agreement with previous reported values derived for HD 49933 and HD 52265. For HD 43587, although we do not detect a rotation period, the timescale of the longer granulation component (15.4 days) is much longer than expected for stellar granulation. Hence, this timescale is probably related with stellar activity, either spot rotational modulation or the timescale of emergence of active regions.

\section{Transit parameter retrieval}

Very few transit analysis studies have included GPs to model stellar variability. The previous GP implementations were so slow that applications to large datasets were almost prohibitive. The exceptions are mostly evolved stars because of their longer variability timescales and higher variability amplitude, for example Kepler-91 (Barclay et al. 2015), Kepler-419 (Dawson et al. 2014), and K2-97b (Grunblatt et al. 2016). These stars were successfully analysed using GPs, but only one GP component was considered. In previous observations with the CoRoT and Kepler satellites, multiple-component noise models were not required due to a combination of the low $\mathrm{S} / \mathrm{N}$ and lower cadence. For example, for the long cadence of Kepler the shorter timescales would be averaged out leaving just the rotational modulation, and possibly the long-period granulation in the light curve. Many methods were used to detrend the rotation modulation in light curves including, albeit rarely, GPs. Moreover, most of the short cadence light curves did not have a high enough $\mathrm{S} / \mathrm{N}$ to detect the small amplitudes of the oscillations and granulation $(<50$ ppm Tables 7 and 6).

As we show in the previous section, for high signal-to-noise observations taken at high cadence (30 s) a larger number of components is needed to correctly account for the variability in the light curves. However, when we are interested in transit parameter retrieval it is more important to determine whether the higher number of components would lead to a more accurate derivation of transit parameters. To test this, we injected planetary transits in the above light curves and derived the transit parameters comparing the one-component model with the best model found in the previous section. For completeness we also compare them with transit parameters derived with a classical non-GP model. For simplicity, for HD 49933 for which we have 
S. C. C. Barros et al.: Improving transit characterisation with Gaussian process modelling of stellar variability

Table 11. Simulated transit parameters for each type of planet.

\begin{tabular}{lccc}
\hline \hline Planet & $T_{0}[\mathrm{~d}]$ & $r_{\mathrm{p}} / R_{\star}$ & $a / R_{\star}$ \\
\hline Jupiter & $t[0]+6$ & 0.07419688 & 18.960539 \\
Neptune & $t[0]+5$ & 0.02581571 & 18.955250 \\
Earth & $t[0]+6$ & 0.00661941 & 18.954969 \\
\hline
\end{tabular}

Notes. We also assumed an orbital period of 15 days, inc $=90^{\circ}$, circular orbits, and quadratic limb darkening coefficients LD1 $=0.5048$ and $\mathrm{LD} 2=0.1468$

two light curves, we considered only the LRa01 light curve because it is longer than IRa01.

\subsection{Transit injection}

In this work we tested a general case of space-based observations coming from transit surveys like CoRoT, Kepler, TESS, or PLATO (Baglin et al. 2006; Borucki et al. 2010; Ricker et al. 2015; Rauer et al. 2014) where several consecutive transits are available. In order to have several transits in our light curves, we chose to inject planets with an intermediate period of 15 days. It will also be interesting to test transits with very short periods and very long periods to test the impact of different regimes of stellar activity in the long term (e.g. rotational and magnetic cycles). Furthermore, it would also be interesting to test observations where just one transit is available, which will be especially relevant for the search of Earth-like planets with TESS or PLATO. However, we leave these analyses of specific cases to future work.

To test different transit signal-to-noise regimes, we injected transits of a Jupiter-size planet (same mass and radius as Jupiter), a Neptune-size planet, and an Earth-size planet. We assumed a Sun-like star and the same quadratic limb darkening coefficients $\mathrm{LD} 1=0.5048$ and LD2 $=0.1468$ for all of the light curves (corresponding to WASP-18 as a random example). We also assumed central transits $\left(\right.$ inc $=90^{\circ}$ ) and circular orbits. For each planet, the simulated normalised separation of the planet $\left(a / R_{\star}\right)$ and the planet-to-star radius ratio $\left(r_{\mathrm{p}} / R_{\star}\right)$ take into account the mass and radius of the planet and the star and the orbital period. The midtransit time was set to be 5 or 6 days after the beginning of the observation of each light curve $t[0]$. The full set of simulated parameters is provided in Table 11. We used the package batman (Kreidberg 2015) to simulate and model the transits.

\subsection{Deriving transit parameters}

To derive the transit parameters of each simulated dataset, we used the best GP noise model, as derived above, or the G1 noise model using the transit model as the mean function. We also compared the performance of the GP models with a non-GP model. We chose a second-order polynomial detrending, which is commonly used in the literature. We extracted the region of the light curves with three times the transit duration and centred in the mid-transit times. Then for each transit we fitted a second-order polynomial to the out-of-transit data and used it to normalise the transit. These normalised transits were fitted simultaneously with an MCMC procedure similar to that explained above, but without the Kernel term, and considering only a white noise component. It should be noted that this procedure changes the data previous to the fit so the comparison with the GP models is not straightforward.
Table 12. Priors for the fitted transit parameters.

\begin{tabular}{lc}
\hline \hline Parameter & Prior \\
\hline$T_{0}$ (days) & $\mathcal{U}(-0.1 ; 0.1)$ \\
$R_{\mathrm{p}} / R_{\star}$ & $\mathcal{U}(0.000001 ; 0.2)$ \\
$a / R_{\star}$ & $\mathcal{J}(1.0 ; 30)$ \\
inc $\left(^{\circ}\right)$ & $\mathcal{S}\left(i_{\text {graz }} ; 90\right)$ \\
\hline
\end{tabular}

Notes. $\mathcal{U}(a ; b)$ is a uniform distribution between $a$ and $b ; \mathcal{J}(a ; b)$ is a Jeffreys distribution between $a$ and $b ; \mathcal{S}(a, b)$ is a sine distribution between $a$ and $b$; graz is the inclination corresponding to an impact parameter equal to 1 for a given $a / R_{\star}$.

When preforming the transit retrieval we kept the limb darkening and the transit period fixed to the injected values and fitted the mid-transit time, planet-to-star radius ratio, normalised separation of the planet, and its orbital inclination. The priors used are given in Table 12. We impose a prior on the inclination to insure that the impact parameter is lower than one and prevent very grazing transits. Allowing grazing transits leads to a high degeneracy between the parameters of the transit and prevents an efficient exploration of the parameter space. Moreover, preventing very grazing transits also allows the transit detection to be estimated by analysing only the significance of the derived planet-to-star radius ratio. The priors for the hyper-parameters of the GP noise model used were the same as in the previous section, but we started the chains close to the best solution found previously to speed up convergence. We found that if we started the chains of the hyper-parameters randomly, as before, the convergence could be very slow, especially for the Earth-size planet, although it eventually reached the same result. When using real data, our procedure can be emulated by first fitting the GP noise model to the out-of-transit data. Alternatively, very long chains are required. For the non-GP model we used the same prior for the white noise component as for the GP model. The above MCMC procedure and selection of chains was used to estimate the parameters and uncertainties.

\subsection{Performance of the retrieval}

For the best GP noise model, for the G1 model, and for the non-GP model, we derived the difference between the estimated parameters and the injected ones. These differences are shown in Fig. 4 for the Jupiter- and Neptune-size planets, and in Fig. 5 for the Earth-size planets. Furthermore, in Fig. 5 we also show the value of the injected planet-to-star radius ratio in order to visually access significant detections.

For the Jupiter-size planet we found that for the GP models all parameters are within $3 \sigma$ of the simulated values. The only exception is the sub-giant HD 179079, where the planet-to-star radius ratio and the inclination are biased for the $\mathrm{G} 1$ model by 3.9 and $3.6 \sigma$, respectively. For the best GP noise model (G2R1O1) the planet-to-star radius ratio is still sightly biased $(2.9 \sigma)$, but all the remaining parameters are well retrieved. The uncertainties are in general slightly larger for the G1 model than for the best GP noise model. In contrast, we found that for the non-GP model the derived planet-to-star radius ratio is biased for all the stars, while the derived transit time is biased for HD 49933 and HD 179079. This is mostly due to the derived errors being much smaller than for the GP models. Hence, the non-GP model leads to biased results in some cases and the best GP noise model leads to more precise and accurate results. 

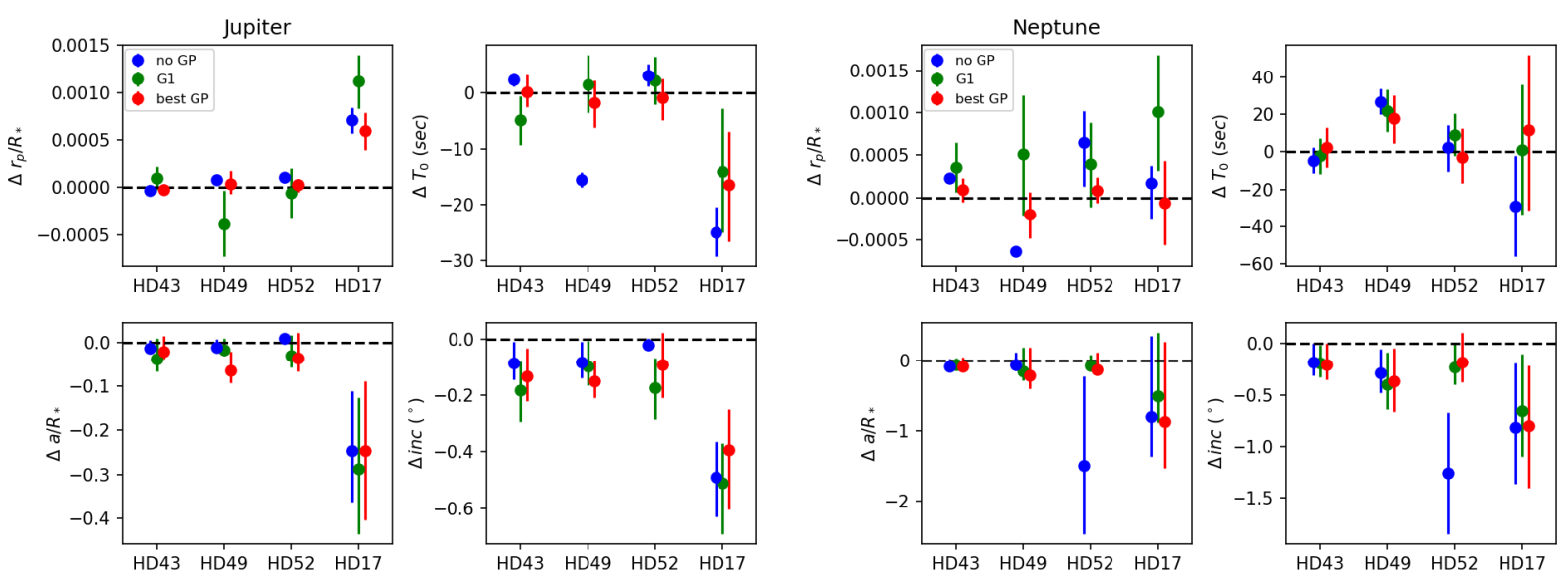

Fig. 4. Difference between the derived and simulated parameters for all fitted parameters. Left: results for the Jupiter-size planet. Right: results for Neptune-size planet. Shown are the G1 model (in green) and the best GP noise model (in red). The true value is shown as a dashed line.
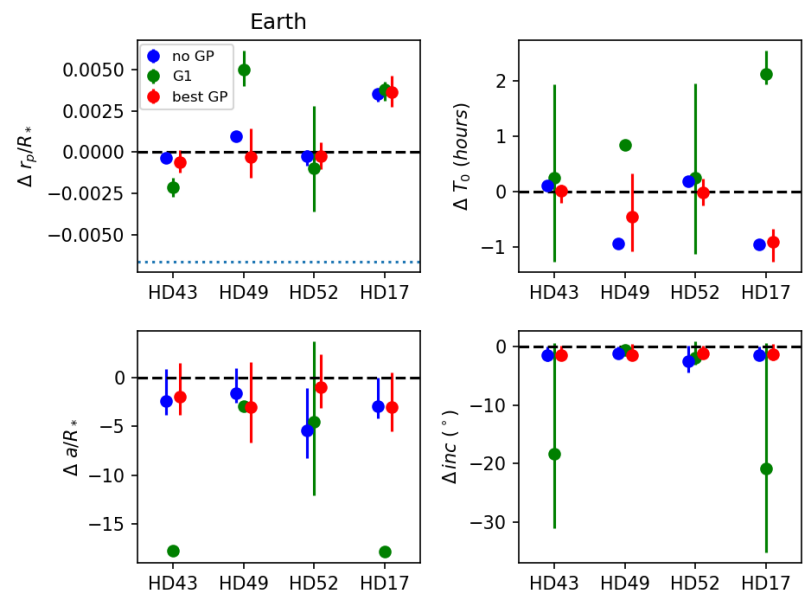

Fig. 5. Difference between the derived and the injected parameters for the Earth-size planet. Shown are the G1 model (in green) and the best GP noise model (in red). The dotted line shows the value of the injected planet-to-star radius ratio (implying that a depth measurement $3 \sigma$ away from this line is consistent with a depth of zero and is therefore not significant). The unit of time is hours, and not seconds as for the larger planets. The true value is shown as a dashed line.

For the Neptune-size planet all the derived parameters of the GP models are within $3 \sigma$ of the injected values. The uncertainties of $R_{\mathrm{p}} / R_{\star}$ are larger for the G1 model than for the best GP noise model, but in general for the other parameters the uncertainties are similar for both models. In contrast, we found that for the non-GP model the derived planet-to-star radius ratio is biased for HD 43587 and HD 49933, and the derived transit time is biased for HD 49933. This is due to the underestimation of the errors in the non-GP model. Hence, the GP models are more accurate than the non-GP model.

For the Earth-size planet the estimated values for the light curve of HD 52265 are within $3 \sigma$ of the injected values for all models except the transit time derived with the non-GP model. However, the derived value of the planet-to-star radius ratio is not significant for the G1 model $\left(r_{\mathrm{p}} / R_{\star}=0.00563_{-0.00261}^{+0.00379}\right)$, while the planet is well retrieved for the best GP noise model and the nonGP model. For the light curve of HD 43587, the G1 model is biased for both $r_{\mathrm{p}} / R_{\star}$ and $a / R_{\star}$. The large mismatch of $a / R_{\star}$ is actually an indication that the G1 model did not find the correct signal of the Earth-size planet and could be, in fact, modelling stellar variability. For the best GP noise model all of the parameters are unbiased and the correct signal of the Earth-size planet was retrieved. For the non-GP model the transit is well retrieved and there is only a bias in the derived transit time. For the light curve of HD 49933 (LRa01), with the G1 model all the parameters are biased (up to $122 \sigma$ ) except the inclination suggesting that the transit was mistaken for stellar activity. Using the best GP noise model all of the parameters are unbiased and the correct signal of the Earth-size planet was found. For the non-GP model the transit is well retrieved, but the planet-to-star radius ratio and the transit time are both biased due to an underestimation of the errors. Finally, for the light curve of the sub-giant HD 179079, the G1 model derived parameters are very biased except the inclination, which is unconstrained. This indicates that the G1 model is strongly biased by stellar variability and the transit signal of the Earth-size planet was not found. The best GP model (G2R1O1) retrieved the transit, but the planet-to-star radius ratio is biased by $4 \sigma$ and the mid-transit time is biased by $3.8 \sigma$. For the non-GP model the planet-to-star radius ratio and the transit time are both biased due to an underestimation of the errors, but the transit is well retrieved. In this case the $a / R_{\star}$ and inc errors are similar to or larger than the best GP model, and they are not biased.

In summary, for the Earth-size planet the G1 model did not detect the planet for HD 52265 and did not find the correct transit signal for the planet for HD 43587, HD 49933, and HD 179079 due to stellar variability. In contrast, the best GP noise model correctly retrieved the Earth-size although for the sub-giant star the parameters are biased. The non-GP model also correctly retrieved the Earth-size although the planet-to-star radius ratio and the mid transit time are biased for some of the stars. Somewhat surprisingly, the non-GP model retrieves the Earth-size planet better than the G1 model, probably because the G1 model provides a poor description of the activity and has more freedom. The method we used for the classical approach changes the data, which can lead to bias, but it also restricts the parameter space and helps constrain the transit. For example, cutting the light curves will not allow the transit solutions found by the G1 model for HD 43587 and HD 179079. Centring the cut light curves in the correct mid-transit time might also help the performance of the non-GP model. Finally, the second-degree polynomial normalisation using the correct mid-transit time also helps the model. For real cases where both the cutting of the light 
curve and the normalisation is done without the knowledge of the correct transit time will lead to further biases in the derived transit parameters. Therefore, we conclude that a better description of the stellar activity is necessary in order to characterise Earth-size planets in high signal-to-noise, high cadence light curves and to favour the best GP model.

\section{Discussion and conclusions}

In this work, we tested whether Gaussian processes allow us to improve the characterisation of transit parameters in cases where stellar variability is the dominant noise source in a light curve. To achieve this goal, we used a sample of five high cadence (32 s), high signal-to-noise observations of four stars taken by the CoRoT satellite. We started by determining which and how many stellar variability components were present in each light curve. We tested models with a maximum of five variability components with a combination of stellar oscillations, granulation, and rotation. Using model comparison, for our sample we found that the best GP noise model requires at least four to five variability components contrasting with the common practice of using only one variability component for transit retrieval. The difference of marginal likelihood between the best model and the one-component model is extremely large, and hence multi-component models are highly favoured.

For light curves with a derived rotation period, we found that the best GP model was composed of one oscillation component, two granulation components, and one rotation component (G2R1O1). For HD 43587, for which we do not constrain the rotational period, we found that the best GP model includes one oscillation component and four granulation components. In this case we attribute the longer timescale granulation component (period $=15.4$ days) to stellar activity without a clear sinusoidal signal (Harvey 1985), and hence it is better described by the granulation kernel.

We found that for the best GP noise model the derived timescales are in qualitative agreement with results from asterosismology. Therefore, our model provides results consistent with our astrophysical knowledge of the star. The advantage of GPs is that the models are defined and applied in the time domain and hence they can be combined easily with a transit model. In this way, GPs can be used to model the stellar variability simultaneously with transit modelling. The alternative is to use a two-step approach where first we filter the stellar variability and then we preform transit modelling. However, for low signal-tonoise transits, filtering the stellar variability can deform or even remove the transits. Therefore, models that couple the transit model with the stellar variability model are needed for planetary characterisation.

For transit analysis, it is more relevant if the number of components used to describe stellar activity affects the derivation of transit parameters. Hence, in the second part of this work we tested whether the best GP model found also improves transit parameter estimation relative to the one-component model. For completeness, we also compared the GP models with a non-GP model. As a non-GP model we chose a second-degree polynomial detrending, which is commonly used in the literature. We recommend avoiding overinterpretation of the comparison between a GP model and a non-GP model because details of the non-GP model chosen can influence the results and the conclusions. Furthermore, non-GP approaches change the data, which can bias the transit model and in our case can help constrain it. We tested a general case of a planet with a 15-day orbital period so that several transits were present in the light curves. We also tested three different planet sizes, Jupiter, Neptune, and Earth, to probe the different signal-to-noise regimes.

For a Jupiter- and Neptune-size planet, the classical seconddegree polynomial detrending leads to a bias in the determination of the planet-to-star radius ratio and the mid-transit time. Accounting for activity with a one-component GP results in larger error bars that unbiases the parameter derivation. Interestingly, when more GP components are added to better describe stellar activity the uncertainty of the parameters slightly decreases and hence the more complex model is more precise while maintaining accuracy. However, for large planets the best GP model is significantly better than the one-component GP model only for the case of the sub-giant HD 179079 because sub-giants have longer timescales of variability and higher amplitudes, which is also why to date it has only been necessary to account for stellar variability for sub-giants and giants (Dawson et al. 2014; Barclay et al. 2015; Grunblatt et al. 2016). Therefore, we conclude that as long as several transits are observed and the S/N of the transit is high (for Jupiter- and Neptune-size planets) a simple GP model is sufficient to correct stellar variability and allows us to derive unbiased planetary parameters.

For the Earth-size planet we found that the non-GP model performs better than the G1 model. This is probably due to the data being modified by the normalisation procedure and because the G1 model is not a good description of the stellar activity. Furthermore, as low signal-to-noise transits can be mistaken for stellar activity and the non-GP model assumes no stellar activity the transit is better retrieved. However, in real data this could lead to false detections if the existence and time of transit is not known a priori. We also found that when including more components in the GP model, the stellar activity is better characterised leading to significantly better results than the one-component model for all the targets. The multi-component GP model allows us to correctly retrieve the transit model (while the one-component model fails), and it leads to more accurate results then the non-GP model. Hence, we conclude that the multi-component model is necessary for low signal-to-noise transits. Therefore, in the case of small planets, we recommend using a multi-component GP model in the transit analysis. A better characterisation of stellar activity leads to a much better planetary characterisation. These results will be relevant for the analysis of transit light curves from TESS, CHEOPS, and PLATO.

We tested here a case of a 15-day orbital period where several transits were obtained. We expect that in other cases, for example very short periods (where the ingress-egress timescale is shorter and similar to the stellar variability timescales), very long periods (due to the low number of transits), or single transits, having a better model for stellar activity will also be important. In future work we plan to explore the advantages and disadvantages of this framework in the characterisation of transiting planets in some of these cases. In particular, small planets with very few available transits (1-2) will be relevant for the search of Earth-like planets with PLATO.

Acknowledgements. S.C.C.B. acknowledges support from Fundação para a Ciência e a Tecnologia (FCT) through Investigador FCT contract IF/01312/2014/CP1215/CT0004. O.D.S.D. is supported in the form of work contract (DL 57/2016/CP1364/CT0004) funded by national funds through FCT. F.P. acknowledges support from fellowship PD/BD/135227/2017 funded by FCT (Portugal) and POPH/FSE (EC) This work was supported by FCT through national funds (PTDC/FIS-AST/28953/2017) and by FEDER - Fundo Europeu de Desenvolvimento Regional through COMPETE2020 - Programa Operacional Competitividade e Internacionalização (POCI-01-0145-FEDER-028953) and through national funds (PIDDAC) by the grant UID/FIS/04434/2019. This publication was written in the framework of the International Team on "Researching 
the Diversity of Planetary Systems" at ISSI (International Space Science Institute) in Bern. We acknowledge the financial support of ISSI and thank them for their hospitality.

\section{References}

Aigrain, S., Hodgkin, S. T., Irwin, M. J., Lewis, J. R., \& Roberts, S. J. 2015, MNRAS, 447, 2880

Appourchaux, T., Michel, E., Auvergne, M., et al. 2008, A\&A, 488, 705

Auvergne, M., Bodin, P., Boisnard, L., et al. 2009, A\&A, 506, 411

Baglin, A., Auvergne, M., Boisnard, L., et al. 2006, in COSPAR, Plenary Meeting, 36th COSPAR Scientific Assembly, 36, 3749

Baliunas, S. L., Donahue, R. A., Soon, W. H., et al. 1995, ApJ, 438, 269

Ballot, J., Gizon, L., Samadi, R., et al. 2011, A\&A, 530, A97

Barclay, T., Endl, M., Huber, D., et al. 2015, ApJ, 800, 46

Barros, S. C. C., Boué, G., Gibson, N. P., et al. 2013, MNRAS, 430, 3032

Barros, S. C. C., Almenara, J. M., Deleuil, M., et al. 2014, A\&A, 569, A74

Bastien, F. A., Stassun, K. G., \& Pepper, J. 2014, ApJ, 788, L9

Benomar, O., Baudin, F., Campante, T. L., et al. 2009, A\&A, 507, L13

Borucki, W. J., Koch, D., Basri, G., et al. 2010, Science, 327, 977

Boumier, P., Benomar, O., Baudin, F., et al. 2014, A\&A, 564, A34

Christian, D. J., Gibson, N. P., Simpson, E. K., et al. 2009, MNRAS, 392, 1585

CoRot Team 2016, The CoRoT Legacy Book: The adventure of the ultra high precision photometry from space, by the CoRot Team (Les Ulis: EDP Sciences)

Corsaro, E., De Ridder, J., \& García, R. A. 2015, A\&A, 579, A83

Cranmer, S. R., Bastien, F. A., Stassun, K. G., \& Saar, S. H. 2014, ApJ, 781, 124

Czesla, S., Huber, K. F., Wolter, U., Schröter, S., \& Schmitt, J. H. M. M. 2009, A\&A, 505, 1277

Dawson, R. I., Johnson, J. A., Fabrycky, D. C., et al. 2014, ApJ, 791, 89

Díaz, R. F., Almenara, J. M., Santerne, A., et al. 2014, MNRAS, 441, 983

Faria, J. P., Haywood, R. D., Brewer, B. J., et al. 2016, A\&A, 588, A31

Foreman-Mackey, D., Agol, E., Ambikasaran, S., \& Angus, R. 2017, AJ, 154 220
Foreman-Mackey, D., Hogg, D. W., Lang, D., \& Goodman, J. 2013, PASP, 125, 306

Geweke, J. 1992, Bayesian Statistic (Oxford: Oxford University Press), 169

Gibson, N. P., Aigrain, S., Roberts, S., et al. 2012, MNRAS, 419, 2683

Gilliland, R. L., Chaplin, W. J., Dunham, E. W., et al. 2011, ApJS, 197, 6

Grunblatt, S. K., Huber, D., Gaidos, E. J., et al. 2016, AJ, 152, 185

Hall, J. C., Henry, G. W., \& Lockwood, G. W. 2007, AJ, 133, 2206

Harvey, J. 1985, ESA SP, 235, 199

Haywood, R. D., Collier Cameron, A., Queloz, D., \& et al. 2014, MNRAS, submitted

Jeffreys, H. 1998, Theory of Probability (Oxford: Oxford University Press)

Kallinger, T., De Ridder, J., Hekker, S., et al. 2014, A\&A, 570, A41

Kass, R. E., \& Raftery, A. E. 1995, J. Am. Stat. Assoc., 90, 773

Kjeldsen, H., \& Bedding, T. R. 1995, A\&A, 293, 87

Kreidberg, L. 2015, PASP, 127, 1161

Lagrange, A.-M., Desort, M., \& Meunier, N. 2010, A\&A, 512, A38

Léger, A., Rouan, D., Schneider, J., et al. 2009, A\&A, 506, 287

Lovis, C., Dumusque, X., Santos, N. C., et al. 2011, ArXiv e-prints [arXiv:1107.5325]

Maciejewski, G., Dimitrov, D., Neuhäuser, R., et al. 2011, MNRAS, 411, 1204

Mathur, S., Hekker, S., Trampedach, R., et al. 2011, ApJ, 741, 119

Meunier, N., Lagrange, A.-M., Borgniet, S., \& Rieutord, M. 2015, A\&A, 583, A118

Nelson, B. E., Ford, E. B., Buchner, J., et al. 2018, AJ, submitted [arXiv:1806.04683]

Pereira, F., Campante, T. L., Cunha, M. S., et al. 2019, MNRAS, 489, 5764

Perrakis, K., Ntzoufras, I., \& Tsionas, E. G. 2014, Comput. Stat. Data Anal., 77, 54

Queloz, D., Bouchy, F., Moutou, C., et al. 2009, A\&A, 506, 303

Rasmussen, C. E., \& Williams, C. K. I. 2006, Gaussian Processes for Machine Learning (Cambridge, MA: MIT Press)

Rauer, H., Catala, C., Aerts, C., et al. 2014, Exp. Astron., 38, 249

Ricker, G. R., Winn, J. N., Vanderspek, R., et al. 2015, J. Astron. Telesc. Instrum. Syst., 1, 014003

Serrano, L. M., Barros, S. C. C., Oshagh, M., et al. 2018, A\&A, 611, A8 